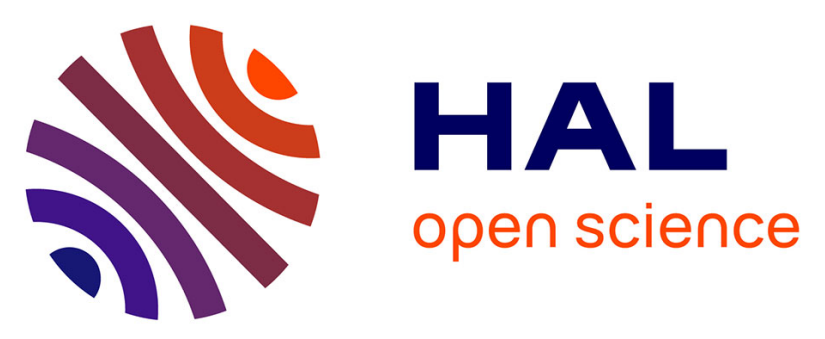

\title{
A numerical method based on Taylor series for bifurcation analyses within Föppl-von Karman plate theory
}

\author{
Haitao Tian, Michel Potier-Ferry, Farid Abed-Meraim
}

\section{- To cite this version:}

Haitao Tian, Michel Potier-Ferry, Farid Abed-Meraim. A numerical method based on Taylor series for bifurcation analyses within Föppl-von Karman plate theory. Mechanics Research Communications, 2018, 93, pp.154-158. 10.1016/j.mechrescom.2017.12.006 . hal-02386528

\section{HAL Id: hal-02386528 \\ https://hal.science/hal-02386528}

Submitted on 29 Nov 2019

HAL is a multi-disciplinary open access archive for the deposit and dissemination of scientific research documents, whether they are published or not. The documents may come from teaching and research institutions in France or abroad, or from public or private research centers.
L'archive ouverte pluridisciplinaire HAL, est destinée au dépôt et à la diffusion de documents scientifiques de niveau recherche, publiés ou non, émanant des établissements d'enseignement et de recherche français ou étrangers, des laboratoires publics ou privés. 


\title{
A numerical method based on Taylor series for bifurcation analyses within Föppl-von Karman plate theory
}

\author{
H. Tian ${ }^{\mathrm{a}}$, M. Potier-Ferry ${ }^{\mathrm{b}}$, F. Abed-Meraim ${ }^{\mathrm{a}}$ \\ ${ }^{a}$ Laboratoire d'Étude des Microstructures et de Mécanique des Matériaux, LEM3, UMR \\ 7239, CNRS, Arts et Métiers ParisTech, 4 rue Augustin Fresnel, 57078, Metz Cedex 3, \\ France \\ ${ }^{b}$ Laboratoire d'Étude des Microstructures et de Mécanique des Matériaux, LEM3, UMR \\ CNRS 7239, Université de Lorraine, 7 rue Félix Savart, BP 15082, 57073, Metz Cedex \\ 3, France
}

\begin{abstract}
A new numerical technique for post-buckling analysis is presented by combining the Asymptotic Numerical Method (ANM) and the Taylor Meshless Method (TMM). These two methods are based on Taylor series, with respect to a scalar load parameter for ANM and with respect to the space variables for TMM. The advantage of ANM is an adaptive step length and this is very efficient near bifurcation points. The specificity of TMM is a quasi-exact solution of the PDEs inside the domain, which leads to a strong reduction of the number of degrees of freedom (DOFs).
\end{abstract}

Keywords:

Taylor meshless, Trefftz method, Asymptotic numerical method, bifurcation, buckling

\section{Introduction}

Many progresses have been made over the last forty years concerning the numerical computation of bifurcation problems. A first way is to solve the "extended system" characterizing the bifurcation points [1]. A simpler technique is to solve a continuation problem with an arc-length control for passing limit points [2]. Nowadays this standard procedure is available in many existing codes, especially in extensively used commercial packages. Note that this continuation technique requires the introduction of a perturbation to capture 
the bifurcation path in a secure manner and a balance between a sufficiently large perturbation and a sufficiently small step length is required for capturing the post-bifurcation response. This difficulty is partially removed with the Asymptotic Numerical Method (ANM), where each step is a Taylor series with respect to a control parameter, see [3] [4], the key point being an adaptive step length related to the radius of convergence of the Taylor series. This adaptivity permits to compute the post-bifurcation curves with a very small perturbation. Moreover, as underlined in [5], one observes an accumulation of small ANM-steps close to a bifurcation point because the radius of convergence is strongly connected with the distance to a neighbor bifurcation point. So an accumulation of small ANM-steps is a simple criteron for detecting bifurcations, but there are other techniques to analyse bifurcation problems within ANM, for instance by computing a bifurcation indicator [6] [7] or by identifying a geometric progression in the computed Taylor series [8]. More generally, ANM is an efficient path following technique able to solve a number of non-linear problems, including unilateral contact or plasticity problems [9].

A discretization method has to be associated with a non-linear solver as ANM and, in most of the cases, this was the finite element method. Nevertheless one can mention several papers where ANM was coupled with a meshless discretization method [10][11][12]. Benefits and drawbacks of meshless techniques are well known and will not be re-discussed here. In this paper, we aim to combine ANM with a meshless method based on Taylor series introduced in [13] and called Taylor Meshless Method (TMM). The latter belongs to the large family of Trefftz methods that use exact solutions of the PDE as shape functions, see for example [14]. The Method of Fundamental Solution (MFS) is likely the most used Trefftz method [15]. The main advantage of Trefftz method and MFS is a strong reduction of the number of unknowns: for instance in [16] a problem was solved with only 90 DOFs while it needs more than 5000 with quadratic finite elements and much more with linear interpolation. The main drawback of Trefftz-type methods is matrix ill-conditioning that prevents solving large-scale problems [17] [18], even if there were many works to try to improve this condition number, see for instance [19][20]. Nevertheless, splitting in subdomains is a simple manner to control the ill-conditioning and several procedures are available [21], what allowed to solve large-scale problems in the Taylor meshless framework [22].

The treatment of non-linear problems is not straightforward within Trefftz methods, because it is not possible to get exact solutions of non-linear or non 
homogeneous problems by inverting the exact tangent operator. Thus, one generally re-introduces a discretization of the domain by radial functions that are combined with fundamental solutions of a reference operator. Typical applications concern Poisson problem [23], newtonian fluids [24] or plasticity [25] and the non-linear problem is solved by Picard iterations, but also by ANM [10] [11]. One can avoid the spatial discretization when using the method of Taylor series, in which case one can moreover obtain accurately the general solution of the homogeneous tangent problem, see the paper [26]. In the latter paper, the polynomial shape functions were computed via an Automatic Differentiation procedure [27], what could permit a wide range of applications.

In the present paper, ANM will be combined with the Taylor meshless method: in other words, we shall perform together Taylor series in space and in load parameter. This double Taylor series expansion will be applied to the famous Föppl-von Karman plate model [28].

\section{Taylor series in loading (ANM)}

The first part of the algorithm is the Asymptotic Numerical Method (ANM) that permits to transform a non-linear PDE into a sequence of linear ones, whose unknowns are the terms of a Taylor series. Here, it will be applied to the Föppl-von Karman equations for elastic plates that is a simple and iconic model in non-linear structural mechanics. Roughly, this section does not differ significantly from previous presentations of ANM, the novelty being the combination of ANM with a new discretization technique. This well-known system is as follows:

$$
\left\{\begin{array}{c}
D \Delta^{2} w-[f, w]=\lambda p \\
\frac{1}{E h} \Delta^{2} f+\frac{1}{2}[w, w]=0
\end{array}\right.
$$

In this equation (1), the two unknown fields are the transversal displacement $w(x, y)$ and the stress function of the in-plane problem $f(x, y)$. The Young modulus is named $E, h$ is the plate thickness, $D=E h^{3} / 12\left(1-\nu^{2}\right)$ is the bending stiffness, $p$ is a reference transversal pressure and $\lambda$ is a scalar load parameter. Often, the external loading is defined through the boundary conditions, which will not be detailed here to save space. The classical bracket operator, which is related to the Gaussian curvature, is defined by 


$$
[b, c]=\frac{\partial^{2} b}{\partial x^{2}} \frac{\partial^{2} c}{\partial y^{2}}+\frac{\partial^{2} b}{\partial y^{2}} \frac{\partial^{2} c}{\partial x^{2}}-2 \frac{\partial^{2} b}{\partial x \partial y} \frac{\partial^{2} c}{\partial x \partial y}
$$

The Asymptotic Numerical Method aims at finding solution paths in the space generated by $\{w(x, y), f(x, y), \lambda\}$ and these paths are represented by Taylor series with respect to a path parameter called " $a$ " :

$$
\left\{\begin{array}{c}
w(x, y) \\
f(x, y) \\
\lambda
\end{array}\right\}=\sum_{K=0}^{N} a^{K}\left\{\begin{array}{c}
w_{K}(x, y) \\
f_{K}(x, y) \\
\lambda_{K}
\end{array}\right\}
$$

In the practice, the initial point of the curve $\left\{w_{0}(x, y), f_{0}(x, y), \lambda_{0}\right\}$ is assumed to be known and of course the series is truncated at a finite order $N$. Many path parameters could be chosen and a complete discussion may be found in [29]. The most used path parameter, inspired by the famous arc-length continuation method [2], leads to a very robust continuation procedure. It is given by

$$
a=\left\langle w-w_{0}, w_{1}\right\rangle+\left\langle f-f_{0}, f_{1}\right\rangle+\left(\lambda-\lambda_{0}\right) \lambda_{1}
$$

where the bilinear form $\langle.,$.$\rangle can be defined in various ways. ANM relies on$ a numerical calculation of the terms of Taylor series (3). With account of the series (3), the system (1) becomes a Taylor series with respect to $a$ and all the terms of this series can be set to zero. At the order $K=1$, one gets a linear system satisfied by $\left\{w_{1}(x, y), f_{1}(x, y), \lambda_{1}\right\}$ and that is nothing but that the tangent system:

$$
\left\{\begin{array}{r}
D \Delta^{2} w_{1}-\left[f_{1}, w_{0}\right]-\left[f_{0}, w_{1}\right]=\lambda_{1} p \\
\frac{1}{E h} \Delta^{2} f_{1}+\left[w_{0}, w_{1}\right]=0 \\
\left\langle w_{1}, w_{1}\right\rangle+\left\langle f_{1}, f_{1}\right\rangle+\lambda_{1}^{2}=1
\end{array}\right.
$$

Note nevertheless that the last equation defining the path parameter is scalar, but not linear and it will be adressed separately. At the generic order $K$, the unknowns $\left\{w_{K}(x, y), f_{K}(x, y), \lambda_{K}\right\}$ are solutions of another linear system involving the same linear operator as in (5): 


$$
\left\{\begin{array}{r}
D \Delta^{2} w_{K}-\left[f_{K}, w_{0}\right]-\left[f_{0}, w_{K}\right]=\lambda_{K} p+g_{K}^{n l} \\
\frac{1}{E h} \Delta^{2} f_{K}+\left[w_{0}, w_{K}\right]=h_{K}^{n l} \\
\left\langle w_{K}, w_{1}\right\rangle+\left\langle f_{K}, f_{1}\right\rangle+\lambda_{K} \lambda_{1}=0
\end{array}\right.
$$

where the right-hand-sides are functions of the unknowns at the previous orders:

$$
g_{K}^{n l}=\sum_{k=1}^{K-1}\left[f_{k}, w_{K-k}\right], \quad h_{K}^{n l}=\frac{1}{2} \sum_{k=1}^{K-1}\left[w_{k}, w_{K-k}\right] / 2
$$

Note that the equations (5)(6) can be solved in a recursive manner.

The solution $\left\{w_{1}(x, y), f_{1}(x, y)\right\}$ of $(5)$ is uniquely defined, when it is completed by consistent boundary conditions, and it depends linearly on $\lambda_{1}$ that is unknown at this level. Hence, this solution can be written in the form $w_{1}(x, y)=\lambda_{1} \hat{w}(x, y), f_{1}(x, y)=\lambda_{1} \hat{f}(x, y)$, where $\{\hat{w}(x, y), \hat{f}(x, y)\}$ is solution of the following system:

$$
\left\{\begin{array}{r}
D \Delta^{2} \hat{w}-\left[\hat{f}, w_{0}\right]-\left[f_{0}, \hat{w}\right]=p \\
\frac{1}{E h} \Delta^{2} \hat{f}+\left[w_{0}, \hat{w}\right]=0
\end{array}\right.
$$

As for the solution of the problem (6) at the generic order $K \geq 2$, it can be expressed as $w_{K}=\lambda_{K} \hat{w}+w_{K}^{n l}, f_{K}=\lambda_{K} \hat{f}+f_{K}^{n l}$, where $\left\{w_{K}^{n l}, f_{K}^{n l}\right\}$ is also solution of a linear problem:

$$
\left\{\begin{aligned}
D \Delta^{2} w_{K}^{n l}-\left[f_{K}^{n l}, w_{0}\right]-\left[f_{0}, w_{K}^{n l}\right] & =g_{K}^{n l} \\
\frac{1}{E h} \Delta^{2} f_{K}^{n l}+\left[w_{0}, w_{K}^{n l}\right] & =h_{K}^{n l}
\end{aligned}\right.
$$

Hence, the ANM-procedure leads to a family of linear systems with variable coefficients $(7)(8)$. This procedure does not differ from the one used in previous ANM papers, where these tangent problems were discretized by finite elements. In the present paper, the systems $(7)(8)$ will be solved by the Taylor Meshless Method.

\section{Taylor series in space (TMM)}

In this part, the Taylor Meshless Method (TMM) is used to solve the linear systems with variable coefficients $(7)(8)$. The principle of TMM is to 
expand the unknown fields in Taylor series and to solve the PDEs in the sense of Taylor series [13]. In [26], the corresponding series were computed with the help of Automatic Differentiation (AD). Here, one takes advantage of the algebraic simplicity of the equations to establish explicitly the needed recurrence formulae, what can avoid additional computation costs due to AD. The two unknowns of (7) are approximated by Taylor series truncated at the order $P$ :

$$
\left\{\begin{array}{c}
w(x, y) \\
f(x, y)
\end{array}\right\}=\sum_{i=0}^{P} \sum_{j=0}^{P-i}\left(x-x_{c}\right)^{i}\left(y-y_{c}\right)^{j}\left\{\begin{array}{c}
w_{i j} \\
f_{i j}
\end{array}\right\}
$$

so that the discrete unknowns are the coefficients $\hat{w}_{i j}, \hat{f}_{i j}$. Of course these Taylor coefficients are related to derivatives. The systems (8) are solved in the same manner.

The equations are also expanded into series up to the order $P-4$ (because we deal with fourth order PDEs). For instance the bending equation in (7) is approximated by

$$
D\left(\Delta^{2} \hat{w}\right)_{i j}-\left[\hat{f}, w_{0}\right]_{i j}-\left[f_{0}, \hat{w}\right]_{i j}=p_{i j}, \quad 1 \leq i+j \leq P-4
$$

Let us now focus on the four Taylor coefficients appearing in the previous equation (10) that combine derivatives and products. The computation of the Taylor coefficients of the bilaplacian is obvious

$$
\begin{aligned}
\left(\Delta^{2} \hat{w}\right)_{i j} & =(i+4)(i+3)(i+2)(i+1) \hat{w}_{i+4, j} \\
& +2(i+2)(i+1)(j+2)(j+1) \hat{w}_{i+2, j+2} \\
& +(j+4)(j+3)(j+2)(j+1) \hat{w}_{i, j+4}
\end{aligned}
$$

as well as the tensor of the second derivatives

$$
\left\{\begin{array}{c}
\partial^{2} \hat{w} / \partial x^{2} \\
\partial^{2} \hat{w} / \partial y^{2} \\
\partial^{2} \hat{w} / \partial x \partial y
\end{array}\right\}=\left\{\begin{array}{c}
(i+2)(i+1) \hat{w}_{i+2, j} \\
(j+2)(j+1) \hat{w}_{i, j+2} \\
(i+1)(j+1) \hat{w}_{i+1, j+1}
\end{array}\right\}
$$

Next, the bracket operator in $(10)(2)$ involves simple products whose derivatives are deduced by using the following formula

$$
(F G)_{i j}=\sum_{k=0}^{i} \sum_{l=0}^{j} F_{k l} G_{i-k, j-l}
$$


As for the transverse force $p$, its derivatives are straightforward when it is constant or linear and they can be obtained by AD in other cases.

A recurrence process is easily deduced from the previous formulae (10)(11) (12)(13), which permits to build a particular solution as well as the whole family of solutions of the associated homogeneous system, i.e. one polynomial $\left\{\breve{P^{w}}(x, y), \breve{P^{f}}(x, y)\right\}$ and $8 P-4$ polynomials $\left\{P_{n}^{w}(x, y), P_{n}^{f}(x, y)\right\}$ for the kernel, see for instance [22] for more details about this computation procedure. Finally the general solution of each problem (7) or (8) is computed in the form

$$
\left\{\begin{array}{l}
w(x, y) \\
f(x, y)
\end{array}\right\}=\left\{\begin{array}{c}
\breve{P^{w}}(x, y) \\
\breve{P}^{f}(x, y)
\end{array}\right\}+\sum_{n=1}^{8 P-4} q_{n}\left\{\begin{array}{c}
P_{n}^{w}(x, y) \\
P_{n}^{f}(x, y)
\end{array}\right\}
$$

This method to solve the linearized PDEs $(7)(8)$ is the original point of the present paper. To complete it, one has to apply the boundary conditions, what can be done with Lagrange multipliers as in [21] or by least-square collocation as in [13][22]. Such piecewise resolutions are quite necessary to solve generic boundary value problems and this will be illustrated in the next section.

The auxiliary problems $(7)(8)$ being solved, one can compute all the terms of the ANM-series (3), after having obtained those of the path parameter $\lambda_{K}$. The latter are easily computed from the definition (4) of the path parameter exactly as in the ANM-literature [3]. Last, we have to define the range of validity of the series (3). In conformity with the basic ANM algorithm, one requires that the last term of the series is small with respect to the first one:

$$
a_{\max }=\left\{\delta \frac{\left\|\left\{f_{1}, x_{1}\right\}\right\|}{\left\|\left\{f_{N}, x_{N}\right\}\right\|}\right\}^{1 /(N-1)}
$$

This leads to adaptive step lengths and this is very important when dealing with bifurcation problems. Indeed, as underlined in [5], the radius of convergence is generally governed by the distance to the nearest bifurcation point. That is why one observes an accumulation of small steps close to the bifurcation: hence, such an accumulation leads to a simple bifurcation criterion by sight. Moreover ANM permits to compute a response curve with a very small perturbation force: one just has to choose a sufficiently small accuracy parameter $\delta$. 


\section{Numerical application and last comments}

In this paper, the method described in the sections 2 and 3 will be assessed with a single example. The difficulty in view of applications to non-linear plate models lies in the handling of the Taylor series described in the previous sections, the account of boundary and interface conditions being about the same as in foregoing papers [21] [22]. Let us consider a square plate, the domain is $[-a / 2, a / 2] \times[-a / 2, a / 2]$, the data are written in non-dimensional form: Young's modulus $E=1$, Poisson's ratio $\nu=0.3$, thickness $h=1$, width $a=2$. The loading is a biaxial compression: $\partial^{2} f / \partial n^{2}=-h \sigma_{x}$. The out-of-plane boundary conditions correspond to a simple support $w=$ $\partial^{2} w / \partial n^{2}=0$

Let us begin with a single domain approach. The parameters of the algorithm are the ANM-degree $N=20$, the spatial degree $P=20$, what corresponds to 156 degrees of freedom and the accuracy parameter $\delta=10^{-8}$ in (15), whose smallness is chosen to ensure the path following for a quasi perfect bifurcation. The boundary conditions are accounted by least-square collocation as in [22], with 240 collocation points. This buckling problem is solved by the method presented here and compared with finite element calculations done with the well established code ANSYS. In both calculations, a small symmetry breaking is needed to compute a bifurcating branch by path following. In ANSYS, this is done by a small modal geometric imperfection measured by the parameter $w_{i m p} / h$ called "scaling factor". In the ANM/TMM-calculation, the small perturbation will be a uniform transversal pressure measured in dimensionless terms by $\bar{p}=p a^{4} / E h^{4}=16 p$.

The obtained bifurcation plots are presented in figures 1 and 2 . They are consistent with the analytic bifurcation stress $\bar{\sigma}_{x}=4 \pi^{2} D / L^{2} h \approx 3.615239$. Within ANSYS, we use a scaling factor of $10^{-4}$ or $10^{-6}$ and, within the present method, a transversal pressure $\bar{p}=10^{-6}$. ANSYS was not able to compute the bifurcating curve with a smaller imperfection, even if we suspect that some experts in non-linear calculation should be able to do such a calculation with a commercial package. Clearly the new technique allowed us to compute the bifurcation plot with a much smaller imperfection, simply by choosing a sufficiently large ANM degree and a sufficiently small accuracy parameter $\delta$.

Next one discusses the convergence with the number of subdomains (hconvergence) and with the degree $P$ of the polynomials (p-convergence). The interface and boundary conditions are accounted by the least-square collo- 


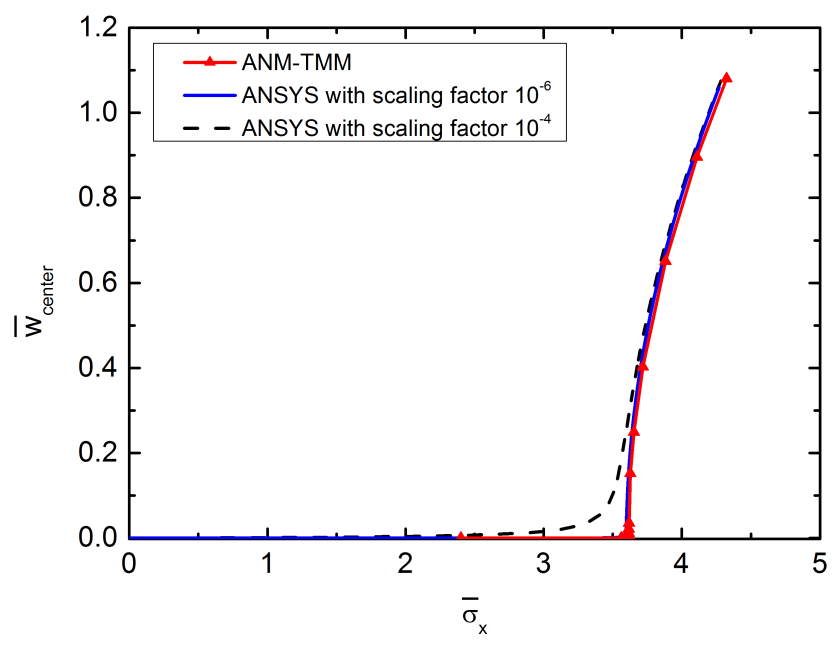

Figure 1: Effect of small perturbations on the buckling of a simply supported square plate. The ANM-TMM algorithm is compared with a commercial finite element code. On the ANM-TMM curve, each point corresponds to one ANM step. The non-dimensional quantities are $\bar{w}=w / h$ and $\bar{\sigma}_{x}=\sigma_{x} a^{2} / E h^{2}$.

cation method in a similar way as [22] [26]. One looks at the value of the bifurcation stress $\bar{\sigma}_{x}$. One has applied degrees $P=5,8,10$ and a number of subdomains varying from 1 to 16 . The results are reported in Figure 3. Clearly the method converges with the degree and/or with the number of subdomains, but good results (i.e. error less than $10^{-3}$ ) are obtained with 9 subdomains and $P \geq 8$ or with 4 subdomains and $P \geq 10$.

So, the new method is very efficient to solve a quasi perfect bifurcation response and this does not require a strong numerical expertise. It seems possible to combine Taylor series in space and in loading parameter, what can be easily extended to other hyperelastic models or to newtonian fluids. This double Taylor series leads to an efficient path following technique. This should be very interesting, for instance in the study of film-substrate systems [30], where large domains are to be considered, in which case the model order reduction by TMM would be very useful. 


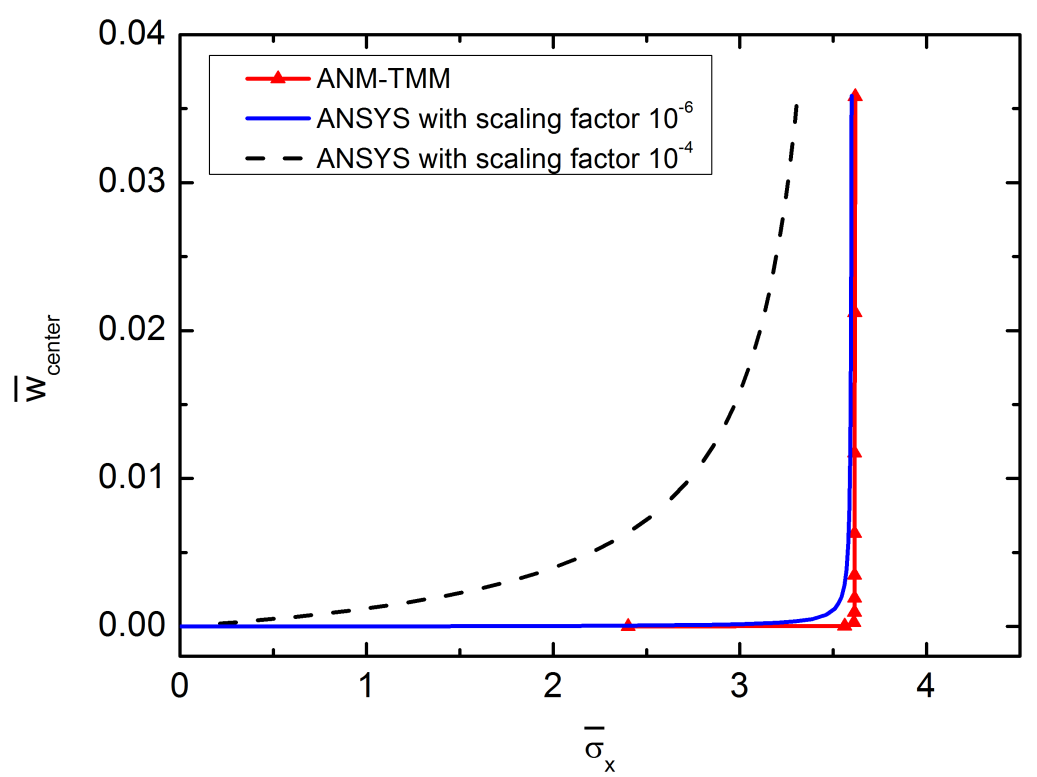

Figure 2: A zoom of Figure 1. One sees that the ANM-TMM method permits to compute easily quasi-perfect bifurcations. On the ANM-TMM curve, each point corresponds to one ANM step.

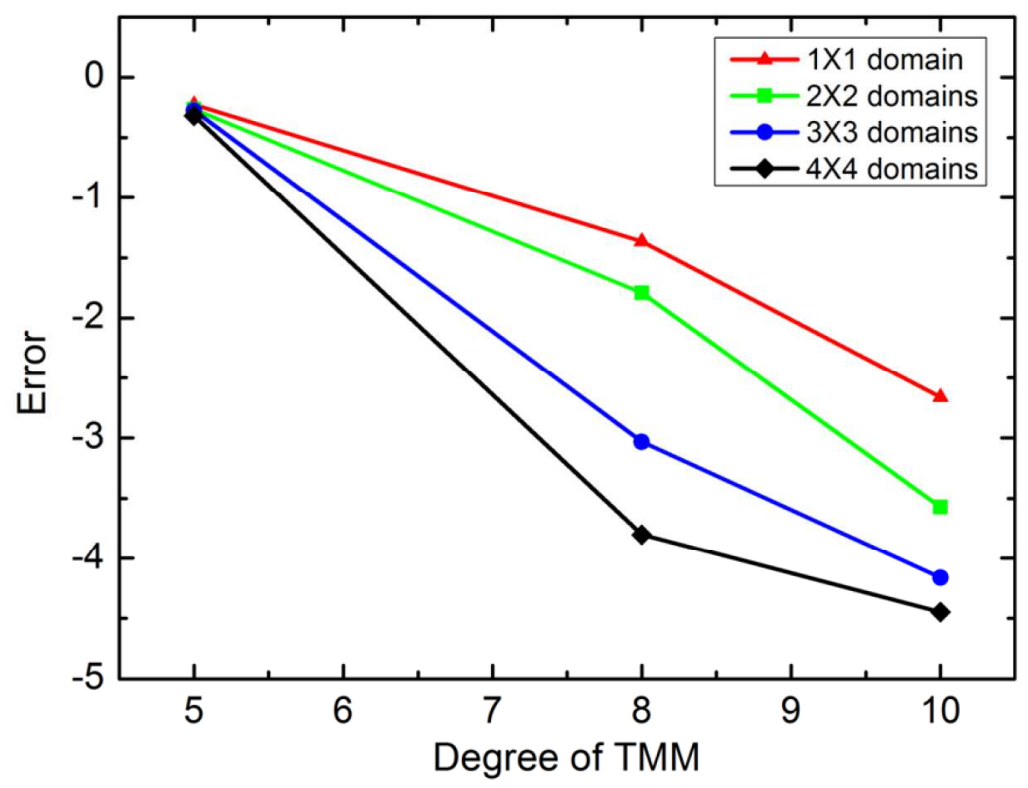

Figure 3: hp-convergence: decimal logarithm of the error on the bifurcation stress, according to the degree $P$ and to the number of subdomains. 


\section{Acknowledgments}

Michel Potier-Ferry dedicates this paper to his long-time and excellent colleague Gérard Maugin. Haitao Tian thanks for his scholarship from the Chinese Science Council. The authors would also like to acknowledge the support of the French government through the National Research Agency ANR (Labex DAMAS, Grant No.ANR-11-LABX-0008-01).

\section{References}

[1] R. Seydel, Numerical computation of branch points in nonlinear equations, Numerische Mathematik 33 (1979) 339-352.

[2] E. Riks, An incremental approach to the solution of snapping and buckling problems, International Journal of Solids and Structures 15 (1979) 529-551.

[3] B. Cochelin, A path-following technique via an asymptotic-numerical method, Computers and Structures 53 (1994) 1181-1192.

[4] B. Cochelin, N. Damil, M. Potier-Ferry, Asymptotic-numerical methods and Padé approximants for non-linear elastic structures, International Journal for Numerical Methods in Engineering 37 (1994) 1187-1213.

[5] S. Baguet, B. Cochelin, On the behaviour of the ANM continuation in the presence of bifurcations, International Journal for Numerical Methods in Biomedical Engineering 19 (2003) 459-471.

[6] E. H. Boutyour, H. Zahrouni, M. Potier-Ferry, M. Boudi, Bifurcation points and bifurcated branches by an asymptotic numerical method and Padé approximants, International Journal for Numerical Methods in Engineering 60 (2004) 1987-2012.

[7] Y. Guevel, H. Boutyour, J. M. Cadou, Automatic detection and branch switching methods for steady bifurcation in fluid mechanics, Journal of Computational Physics 230 (2011) 3614-3629.

[8] B. Cochelin, M. Medale, Power series analysis as a major breakthrough to improve the efficiency of Asymptotic Numerical Method in the vicinity of bifurcations, Journal of Computational Physics 236 (2013) 594607. 
[9] M. Potier-Ferry, N. Damil, B. Braikat, J. Descamps, J. M. Cadou, H. L. Cao, A. Elhage-Hussein, Traitement des fortes non-linéarités par la méthode asymptotique numérique, Comptes Rendus de l'Académie des Sciences, Séries IIB 324 (1997) 171-177.

[10] A. Tri, H. Zahrouni, M. Potier-Ferry, High order continuation algorithm and meshless procedures to solve nonlinear Poisson problems, Engineering Analysis with Boundary Elements 36 (2012) 1705-1714.

[11] A. Tri, H. Zahrouni, M. Potier-Ferry, Bifurcation indicator based on meshless and asymptotic numerical methods for nonlinear Poisson problems, Numerical Methods for Partial Differential Equations 30 (2014) 978-993.

[12] A. Timesli, B. Braikat, H. Lahmam, H. Zahrouni, A new algorithm based on Moving Least Square method to simulate material mixing in friction stir welding, Engineering Analysis with Boundary Elements 50 (2015) 372-380.

[13] D. S. Zézé, M. Potier-Ferry, N. Damil, A boundary meshless method with shape functions computed from the PDE, Engineering Analysis with Boundary Elements 34 (2010) 747-754.

[14] J. A. Kolodziej, A. P. Zieliński, Boundary Collocation Techniques and their Application in Engineering, WIT press, Southampton, 2009.

[15] G. Fairweather, A. Karageorghis, The method of fundamental solutions for elliptic boundary value problems, Advances in Computational Mathematics 9 (1998) 69-95.

[16] Y. Tampango, M. Potier-Ferry, Y. Koutsawa, S. Belouettar, Convergence analysis and detection of singularities within a boundary meshless method based on Taylor series, Engineering Analysis with Boundary Elements 36 (2012) 1465-1472.

[17] R. Schaback, Error estimates and condition numbers for radial basis function interpolation, Advances in Computational Mathematics 3 (1995) 251-264. 
[18] A. H. D. Cheng, M. A. Golberg, E. J. Kansa, G. Zammito, Exponential convergence and Hc multiquadric collocation method for partial differential equations, Numerical Methods for Partial Differential Equations 19 (2003) 571-594.

[19] C. J. Alves, On the choice of source points in the method of fundamental solutions, Engineering Analysis with Boundary Elements 33 (2009) $1348-1361$.

[20] P. Antunes, Reducing the ill conditioning in the method of fundamental solutions, Advances in Computational Mathematics in press. doi:10.1007/s10444-017-9548-6.

[21] Y. Tampango, M. Potier-Ferry, Y. Koutsawa, S. Tiem, Coupling of polynomial approximations with application to a boundary meshless method, International Journal for Numerical Methods in Engineering 95 (2013) 1094-1112.

[22] J. Yang, H. Hu, M. Potier-Ferry, Solving large scale problems by Taylor Meshless Method, International Journal for Numerical Methods in Engineering 112 (2017) 103-124.

[23] K. Balakrishnan, P. A. Ramachandran, Osculatory interpolation in the method of fundamental solution for nonlinear Poisson problems, Journal of Computational Physics 172 (2001) 1-18.

[24] D. Nath, M. S. Kalra, P. Munshi, One-stage Method of Fundamental and Particular Solutions (MFS-MPS) for the steady Navier-Stokes equations in a lid-driven cavity, Engineering Analysis with Boundary Elements 58 (2015) 39-47.

[25] M. Jankowska, J. Kolodziej, A study of elastic-plastic deformation in the plate with the incremental theory and the meshless methods, Journal of Mechanics of Materials and Structures 11 (2016) 41-60.

[26] J. Yang, H. Hu, Y. Koutsawa, M. Potier-Ferry, Taylor meshless method for solving non-linear partial differential equations, Journal of Computational Physics 348 (2017) 385-400. 
[27] A. Griewank, A. Walther, Evaluating derivatives: principles and techniques of algorithmic differentiation, Society for Industrial and Applied Mathematics, Philadelphia, 2008.

[28] P. G. Ciarlet, A justification of the von karman equations, Archive for Rational Mechanics and Analysis 73 (1980) 349-389.

[29] H. Mottaqui, B. Braikat, N. Damil, Discussion about parameterization in the asymptotic numerical method: application to nonlinear elastic shells, Computer Methods in Applied Mechanics and Engineering 199 (2010) 1701-1709.

[30] F. Xu, M. Potier-Ferry, S. Belouettar, Y. Cong, 3D finite element modeling for instabilities in thin films on soft substrates, International Journal of Solids and Structures 51 (2014) 3619-3632.

[31] Z. C. Li, T. T. Lu, H. T. Huang, A. H. D. Cheng, Trefftz, collocation, and other boundary methods: a comparison, Numerical Methods for Partial Differential Equations 23 (2007) 93-144. 